\title{
Chaotic Rotation of a Towed Elliptical Cylinder
}

\author{
G. D. WEYMOUTH \\ Southampton Marine and Maritime Institute, University of Southampton, Southampton, UK
}

(Received 19 November 2013)

In this paper I consider the self-excited rotation of an elliptical cylinder towed in a viscous fluid as a canonical model of nonlinear fluid structure interactions with possible applications in the design of sensors and energy extraction devices. First, the self-excited ellipse system is shown to be analogous to the forced bistable oscillators studied in classic chaos theory. Next, fully coupled computational fluid dynamics simulations of the motion of the cylinder demonstrate limit cycle, period doubling, intermittently chaotic, and fully chaotic dynamics as the distance between the pivot and the centroid is varied. The viscous wake behind the cylinder is presented for the limit cycle cases and new types of stable wakes are characterized. The wake in the chaotic case demonstrates a strong history effect, with a variety of wake types possible for a given structural state. The rotational kinetic energy and fluid vorticity is found to be maximum in a stable period-2 limit cycle associated with leading edge vortices. The adjacent chaotic case has a broadband frequency response and the second highest maximum kinetic energy, 15 times greater than the average over the trajectory. The chaotic response of the system is found to persist when moderate structural damping is applied and down to Reynolds numbers as low as 200 .

Key words: nonlinear dynamics, fluid structure interaction, unsteady fluid mechanics

\section{Introduction}

The analysis of fluid structure interactions (FSI) is fundamental to diverse engineering fields with examples ranging from the flow induced motions of telephone wires and offshore drilling risers (Bearman 1984; Williamson \& Govardhan 2004), to the flutter of membranes such as the wheezing of the soft pallet (Huang 1995) or a flapping flag (Connell \& Yue 2007). Exciting new developments in the field include the design of energy extraction devices which utilize resonant structural response to the flow (Bernitsas et al. 2008; Abdelkefi et al. 2013; Barrero-Gil et al. 2010) and passive hydrodynamic sensors such as those of Beem et al. (2013) which are inspired by the ability of a hunting harbour seal to detect its prey's wake minutes after its passing.

While some of these systems are approximately linear and many feature periodic responses, the non-periodic response of nonlinear deterministic systems have been a source of fascination and rewarding research since their discovery by Lorenz (1963). Holmes (1979) and others developed this theory to apply to a broad range of harmonically forced model systems useful in mechanical applications. Chaotic FSI gives rise to many practical problems such as snap loading (Connell \& Yue 2007), buckling of wings in aircraft manoeuvres (Sipcic 1990) and ship roll and capsize (Spyrou \& Thompson 2000). The broad

\footnotetext{
$\dagger$ Email address for correspondence: G.D.Weymouth@soton.ac.uk
} 
frequency response of chaotic mechanical systems is also important, with its impact on the fatigue life of structures (Modarres-Sadeghi et al. 2011; Dahl et al. 2007), and its ability to maximize response of sensors and energy extraction devices over a broad range of excitation frequencies (Arrieta et al. 2010; Townsend \& Shenoi 2013).

In this paper I consider the self-excited rotation of an elliptical cylinder towed through a viscous fluid as a canonical model of nonlinear fluid structure interactions. Like a simple pendulum, this is a nearly trivial one degree of freedom mechanical system, yet its behaviour is incredibly rich; sensitively ranging through periodic, semi-periodic, and fully chaotic dynamics. The investigation of simple nonlinear FSI systems is an active one and recent experimental work has shown that a single degree of freedom pendular disk in a cross flow exhibits bistability (Obligado et al. 2013). An entire field of literature exists on the rotational galloping and torsional flutter of bluff cylinders due to their prevalence in civil and industrial engineering (Nakamura 1990; Van Oudheusden 1996; Robertson et al. 2003; Alonso et al. 2010). Understandably these analyses are mostly focused on preventing such large amplitude motions and generally feature quasi-static analysis and static experiments at high Reynolds number. In contrast, the present study is focused on describing the nonlinear dynamics of this system free from structural damping or restoring forces. Additionally, the current study uses low Reynolds number $\left(R e \leq 10^{3}\right)$ both because this is more appropriate for small scale flow sensors, but also because it avoids turbulent fluid forcing confusing the analysis of this deterministic system. Another related area of research is that of auto-rotation (Lugt 1983), in which an object such as a flat plate rotates about an axis perpendicular to an oncoming flow despite having a nominally zero net torque about its center. The rotation is due to the unsteady vortex shedding, and while thick ellipses show much less tendency to auto-rotated than plates due to their rounded edges they are near the stability limit (Lugt 1980).

The towed rotating ellipse is a uniquely simple example of chaotic FSI. While the fluid supplies infinitely many degrees of freedom and nonlinear forcing even at low Reynolds number, previous example systems depend strongly on continuously deformable or multilinkage structures or on turbulent flow. Indeed, the onset of chaotic motions in flexible risers is attributed to the interaction of structural waves and fluid gradients along the span (Modarres-Sadeghi et al. 2011). And flapping membranes with excessive stiffness or reduced mass-ratio exhibit only simple period-1 oscillations up to $R e=5000$ (Connell \& Yue 2007). In the one degree of freedom rotational galloping experiments of Van Oudheusden (1996) two stable limit cycles are found for certain parameters, but the author explains the difficulty in isolating this possible bifurcation experimentally. The current work is therefore not only beneficial because of the problem's relevance to engineering systems, but also because of its simplicity and the possibility for detailed investigation using fully coupled computational simulations.

The formulation of the system is detailed in $\S 2$, using analytic arguments to demonstrate its similarity to the classic forced bistable oscillators studied in the early development of chaos theory. Next, $\S 3$ presents the numerical approach used to study the fully coupled unsteady fluid-structure system. In $\S 4$ the trajectories, wake modes, and energy levels are presented over a range of geometric, structural damping, and Re conditions. Finally, $\S 5$ discusses the findings and presents conclusions.

\section{Formulation and Equivalence to Forced Bistable Oscillators}

Consider a two-dimensional elliptical cylinder, towed at steady velocity $U$ through a fluid of density $\rho$, and free to pivot about the tow point. The system is sketched in Fig 1a. The major axis of the ellipse is $L$, the minor axis is $t$, and the instantaneous angle of 
(a)

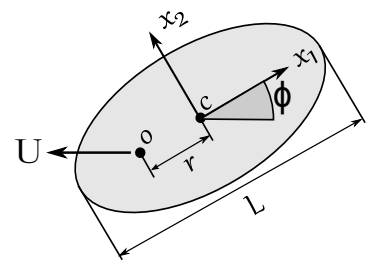

(b)

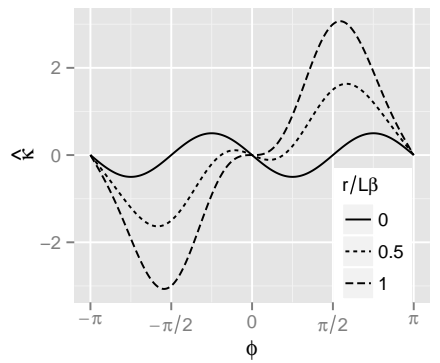

(c)

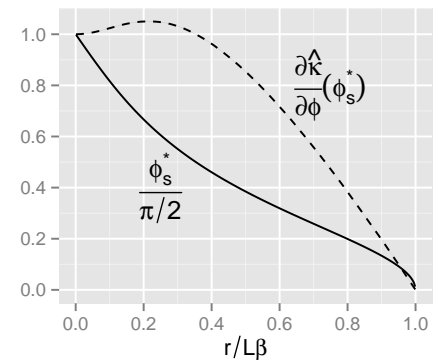

Figure 1: (a) Sketch of the fluid structure interaction problem. The ellipse is towed at constant speed and allowed to rotate freely around the pivot at $o$. (b) Approximate normalized restoring torque $\hat{\kappa}$ given by equation 2.5. (c) Stable fixed point $\phi_{s}^{*}$ and linear spring coefficient at that point $\left.\frac{\partial \hat{\kappa}}{\partial \phi}\right|_{\phi_{s}^{*}}$ as a function of $r / L \beta$.

rotation is $\phi$. The pivot point is located a distance $r$ from the centroid of the ellipse along the major axis.

While the structure has only one degree of freedom, the dynamical system depends on $\phi, \dot{\phi}$, and the state of the continuous fluid. The angular equation of motion about the pivot governs the rotation of the ellipse,

$$
I_{o} \alpha=\tau_{o}=\oint_{S} \vec{f}(s) \times\left(\vec{x}(s)-\vec{x}_{o}\right) d s
$$

where $I_{o}$ is the second moment of area of the ellipse about the pivot, $\alpha=\ddot{\phi}$ is the angular acceleration, and $\tau_{o}$ is the integrated torque relative to the pivot due to the contact force $\vec{f}$ on the solid/fluid interface $S$. The resulting position and velocity of the ellipse set the boundary conditions on the fluid, leading to a fully coupled FSI problem. Note that only the fluid forces are considered; there are no structural damping or restoring torques.

The fluid motion and resultant forces in Eq 2.1 are governed by the full viscous NavierStokes equation, but it is insightful to approximate these forces analytically where possible. In particular, given that the added mass matrix for the ellipse is diagonal, the potential flow estimate is,

$$
\begin{aligned}
\tilde{\tau}_{o} & =-\left(m_{3}+r^{2} m_{2}\right) \alpha+\frac{1}{2} U^{2} \sin 2 \phi\left(m_{2}-m_{1}\right) \\
& =-I_{a} \alpha-\kappa_{M}(\phi)
\end{aligned}
$$

where $m_{i}=\frac{1}{4} \rho \pi\left(t^{2}, L^{2}, \frac{1}{32}\left(L^{2}-t^{2}\right)^{2}\right)$ are the added mass components in each body fixed coordinate direction $\left(x_{1}, x_{2}, x_{3}=\phi\right)$ as labelled in Fig 1a. Equation 2.2 is composed of an angular fluid inertia term $I_{a}$ from the acceleration of the off-center ellipse, and the well known Munk moment $\kappa_{M}(\phi)$ which tends to rotate slender bodies broadside to the flow. Indeed the stable fixed points for this system are clearly $\phi_{s}^{*}= \pm \frac{\pi}{2}$ while the unstable fixed points are $\phi_{u}^{*}=0, \pi$.

Note that the Munk moment has no dependence on the lever arm $r$. In thin airfoil theory the Munk moment is supplemented with the torque induced by the lift force, but this cannot be applied the flow around a bluff body. A simple drag force model,

$$
D=\frac{1}{2} C_{D}(\phi) \rho U^{2} L
$$


is more appropriate, where the drag coefficient could be roughly estimated as $C_{D}=$ $1-\frac{1}{2} \cos 2 \phi$. Additionally, the lack of pressure recovery on the back half of the ellipse which causes the drag will also reduce the magnitude of the Munk moment (Lugt 1980). Applying this drag force to the centroid of the ellipse gives the estimated total restoring torque as,

$$
\hat{\kappa}=\frac{\kappa}{\frac{\beta}{4} \rho U^{2} L^{2}}=-\sin \phi\left[\cos \phi-\frac{r}{L \beta}(2-\cos 2 \phi)\right]
$$

where $\beta$ is an empirical coefficient scaling the Munk moment. When the pivot is at the center the torque is given soley by the Munk moment and the fixed points are located at $\phi_{s}^{*}= \pm \frac{\pi}{2}$, as before. Increasing the size of the lever arm increases the drag component of the restoring torque, driving the stable fixed points toward $\phi=0$ (Fig 1b,1c). At $r / L \beta=1$, the two stable points merge at $\phi=0$ and this fixed point undergoes a Hopf bifurcation and becomes the sole stable fixed point.

The arrangement of two stable fixed points straddling an unstable fixed point classifies the system as a bi-stable oscillator. The classic bistable model system considered in Holmes (1979) is

$$
\ddot{\phi}+\zeta \dot{\phi}-c_{1} \phi+c_{2} \phi^{3}=f \cos (\omega t)
$$

and informative comparisons can be made to this system. Performing a Taylor expansion of Eq 2.5 around $\phi=0$ recovers $\hat{\kappa}=-c_{1} \phi+c_{2} \phi^{3}+O\left(\phi^{5}\right)$ where $c_{1}=1-r / L \beta$ and $c_{2}=\frac{11}{6} r / L \beta+\frac{2}{3}$. The system has a cubic restoring term, which leads to its bi-stability, as in Eq 2.6. However, the nonlinearity in the rotating ellipse system is more severe. First, the polynomial coefficients of the ellipse restoring torque are a function of $\phi$. The linear coefficient of the spring at the stable fixed point, $\left.\frac{\partial \hat{\kappa}}{\partial \phi}\right|_{\phi_{s}^{*}}$, varies strongly with $r / L \beta$ and has a local maximum at $r / L \beta \simeq 0.2$ (Fig 1c) which can not be modeled by Eq 2.6. Second, while the motion through a viscous fluid will supply damping, that damping is nonlinear and depends on both $\phi$ and $\dot{\phi}$. Finally, time-dependent forcing similar to that in Eq 2.6 is supplied by the alternating shedding of vortices off the body, but this will not produce a constant amplitude harmonic force. Instead, the amplitude and timing depend on the state of the near-field flow around the body. These additional nonlinearities require the use of a numerical approach to fully quantify the response of the towed ellipse.

\section{Numerical Methodology for the Fully Coupled Simulations}

A set of two-dimensional simulations enable detailed quantitative predictions of the coupled viscous fluid / dynamic body system. In this work I utilize the Boundary Data Immersion Method (BDIM), a robust immersed boundary method suitable for dynamic fluid-structure interaction problems detailed in Weymouth et al. (2006) and Weymouth \& Yue (2011). Briefly, the full Navier-Stokes equations and the angular governing equation 2.1 are convolved with a kernel of support $\epsilon=2 h$, where $h$ is the grid spacing. The integrated equations are valid over the complete domain and allow for general solid body dynamics to be simulated. Previous work has validated this approach for a variety of dynamic rigid-body problems such as flapping and translating foils (Wibawa et al. 2012) and deforming body problems such as a model of a fast escaping octopus (Weymouth \& Triantafyllou 2013).

The tests are run using a 2:1 ellipse and the body density matches the fluid density. An inertial computational domain is used with dimensions $8 L \times 5 L$ which translates with the body but does not rotate. All cases use the no-slip and no-penetration boundary conditions on the solid/fluid interface. No-penetration conditions are applied on the top 
Table 1: Amplitude of oscillation $\Phi$ and percentage error relative to the finest solution $\epsilon=1-\Phi / \Phi_{h=0.01 L}$ for the $r=0$ period-1 limit cycle presented in $\S 4$.

\begin{tabular}{cccccc}
\hline \hline$h / L$ & 0.039 & 0.026 & 0.02 & 0.013 & 0.01 \\
\hline$\Phi$ & 0.421 & 0.470 & 0.497 & 0.504 & 0.507 \\
$\epsilon$ & 0.17 & 0.074 & 0.020 & 0.007 & - \\
\hline
\end{tabular}

and bottom walls and a convection exit condition is used. This narrow domain incurs blockage effects but tests with larger computational domains indicated that the same nonlinear response features are found, just at slightly different values of the pivot location.

The coupled BDIM equations are discretized using a finite-volume method (third-order convection and second-order diffusion) in space and Heun's explicit second-order method in time. An adaptive time-stepping scheme is used to maintain stability. Table 1 presents a grid convergence study on the magnitude of oscillation for a periodic case with $r=0$. The results converge with third-order accuracy overall and the maximum difference in the solution between the fine and intermediate grid $(h / L=0.02)$ is only $2 \%$. This verifies the high accuracy of these viscous two-dimensional simulations and the intermediate grid level is used for the remainder of the paper.

\section{Results for the Freely Rotating Ellipse}

This section details the numerical results of the self-excited rotations of a 2:1 elliptical cylinder. The Reynolds number based on the steady tow speed $U$ is set to $R e=\frac{U L}{\nu}=10^{3}$ unless otherwise mentioned to avoid intrinsically non-periodic forcing due to transition to turbulent flow on the cylinder. The length of the lever are was systematically varied in the range of $0 \leq r / L \leq \frac{1}{2}$ which simultaneously adjusts the fluid restoring torque and moment of inertia as detailed in $\S 2$. None of the cases studied with the 2:1 ellipse produced fixed point motion due to the spontaneous onset of bluff body vortex shedding. Instead, the trajectories show either limit-cycle, intermittently periodic, or fully chaotic motion.

Figure 2 shows the vortex wake and Fig 3 the time history, power spectral density, and phase portraits of the rotation angle $\phi$ for values of $r / L$ which result in limit cycle trajectories. By combining the structural state information in Fig 3 with the fluid state information in Fig 2, a fair understanding of the system in these simple cases is achieved. These figures have been oriented such that the trajectories all move to the positive fixed point, but adjusted initial conditions make either branch accessible.

When the lever arm is large, the torque induced by the bluff-body drag limits the motions to small amplitude and speed. At $r / L=0.5$ the limit cycle is centered on $\phi=0$ suggesting that this is a stable fixed point. As $r / L$ is decreased the Hopf bifurcation in Eq 2.5 is crossed some time before $r / L=0.4$, destabilizing $\phi=0$ and creating two stable fixed points on either side. This indicates that the value of $\beta$ is approximately $1 / 2$ for this Reynolds number.

There is near-periodic orbit at $r / L=0.4$ with a wake characterized by a standard Karman vortex street. In the literature of vortex induced vibrations (Williamson \& Govardhan 2004) this is categorized as a $2 \mathrm{~S}$ wake, having two single vortices per cycle. Before $r / L=0.3$, the trajectories undergo period doubling as seen clearly in the frequency content of $\phi$. This wake (not shown) features two pairs of vortices, a $2 \mathrm{P}$ wake, 


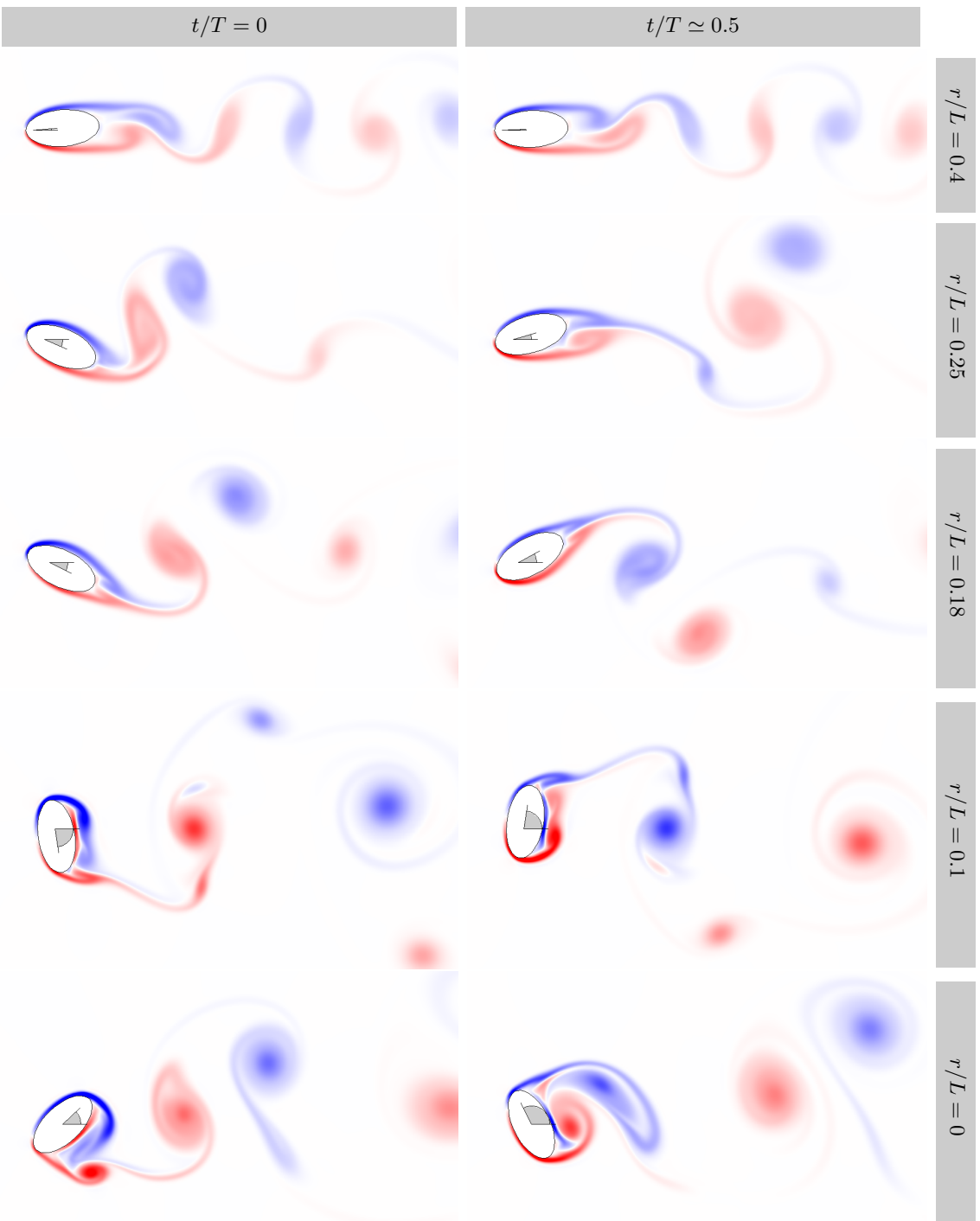

Figure 2: Vorticity field (red/blue) in the wake of the ellipse (black outline, travelling right-to-left) as it rotates freely in limit cycle motion at $R e=1000$. Two times are shown for each value of $r$ at approximately the extreme values of $\phi$. The instantaneous value of $\phi$ is indicated on the pivot of the ellipse for reference.

with one larger than the other. A second period-2 limit-cycle appears at $r / L=0.25$ which has $20 \%$ larger amplitude and a dominant period-2 component. The wake for this cycle is similar, but one of the vortices in the smaller pair is grouped with the larger pair leaving the other small vortex isolated, making this a $\mathrm{T}+\mathrm{S}$ wake (Triplet plus Single).

The region from $0.25>r / L>0.15$ features mostly non-periodic trajectories, but a small amplitude period- 1 limit cycle centered on $\pi / 8$ is found at $r / L=0.2$ with an asymmetric $\mathrm{P}$ wake. A periodic response is also found at $r / L=0.18$ with a large period-3 limit cycle which alternates between the positive and negative fixed points. The wake is a symmetric version of the $r / L=0.25$ limit cycle, with a $2 \mathrm{P}+2 \mathrm{~S}$ wake. This is the first 
(a)

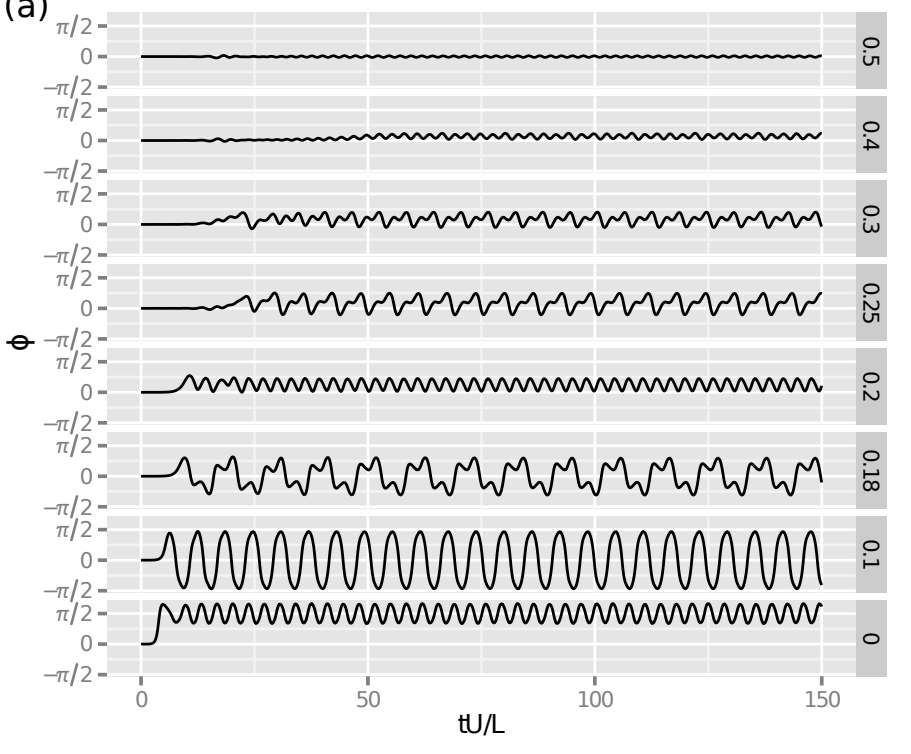

(b)

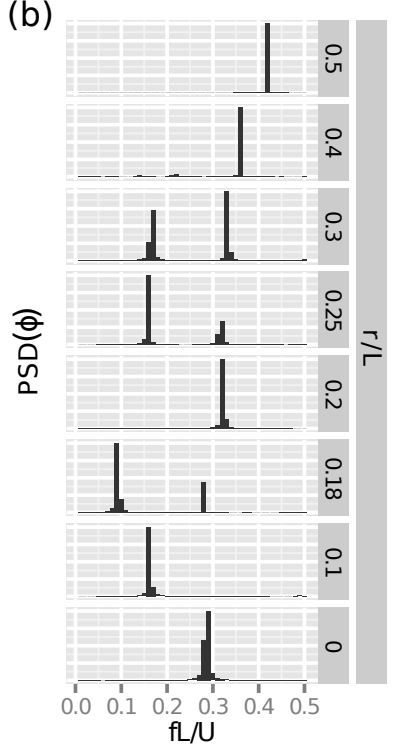

(c)

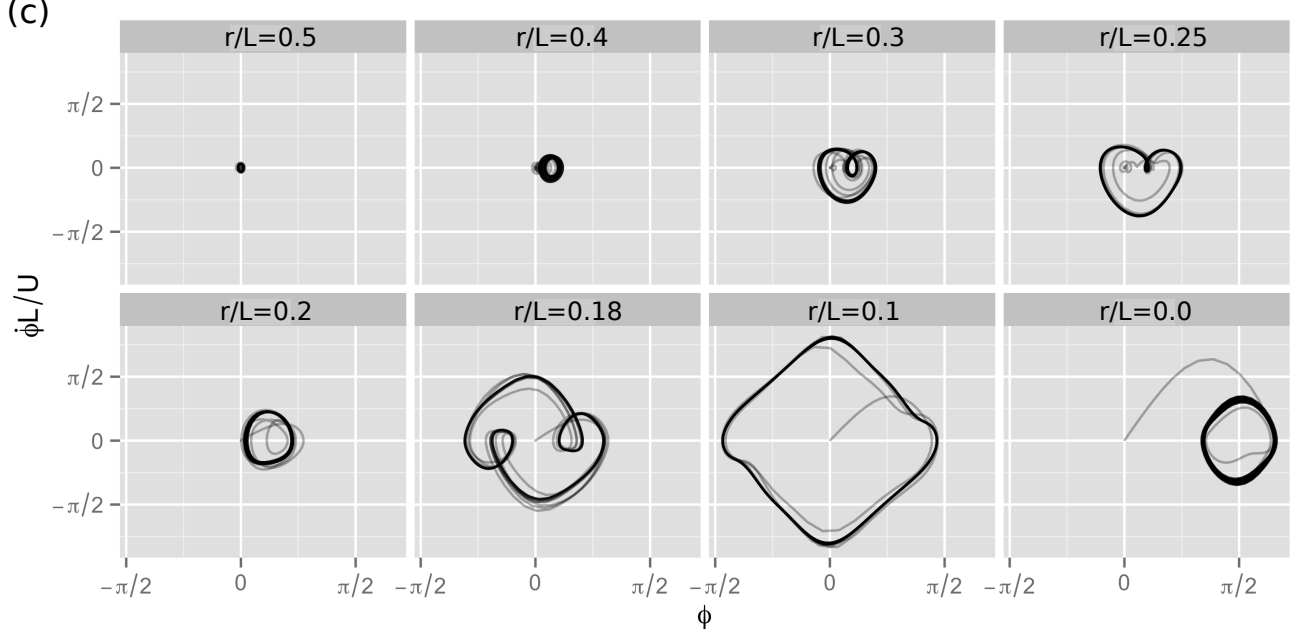

Figure 3: (a) Time history, (b) normalized power spectral density, and (c) phase portraits of $\phi$ for values of $r / L$ which produce periodic limit cycles at $R e=1000$. The phase portraits are drawn with $70 \%$ transparency so that overlapping orbits appear darker.

wake which features leading edge vortex (LEV) separation and these vortices power the trajectory through the unstable fixed point at $\phi=0$.

A stable limit cycle is found between $0.15>r / L>0.05$ with large amplitude $(\Phi \simeq \pi / 2)$ and high velocity $\left(\dot{\Phi} \simeq \frac{3}{4} \pi U / L\right)$ as in rotational galloping. The frequency content shows that this a period-2 limit cycle with nearly zero period- 1 component. LEVs form a vortex street on the centerline with peak vorticity levels double that of the $r / L=0.1$ limit cycle wake. Smaller trailing edge vortices form off-center between the LEVs resulting in a $4 \mathrm{~S}$ wake. As $r / L \rightarrow 0$ this large amplitude orbit decays intermittently into a smaller period-1 cycle centered on $\phi= \pm \pi / 2$ with an asymmetric $\mathrm{P}$ wake.

Overall, the vortex shedding frequency is completely dominant for the limit cycle cases. 
a)

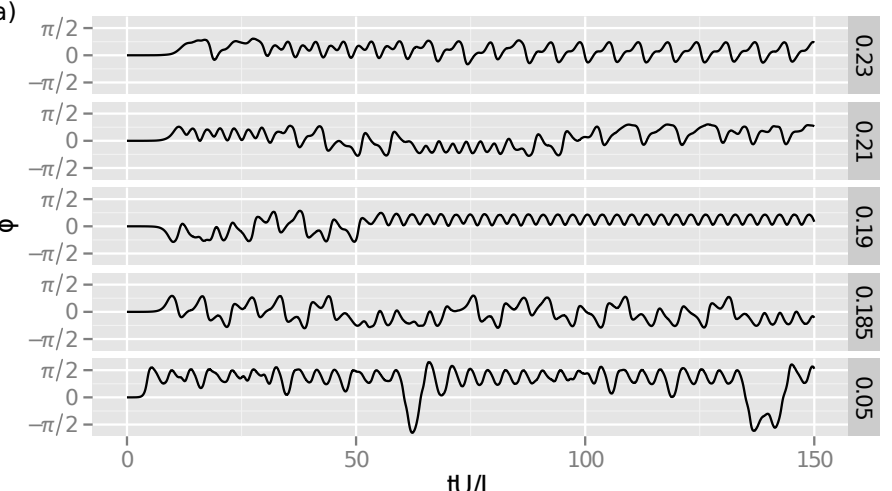

b)

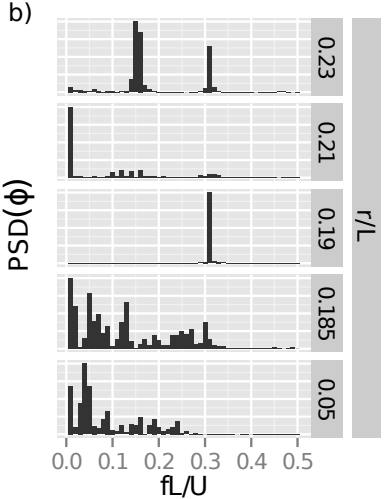

Figure 4: (a) Time history and (b) normalized power spectral density of $\phi$ for values of $r / L$ which produce orbits with intermittently periodic behaviour at $R e=1000$.

The number of vortices corresponds directly to the period of the cycle; two for the period1 cycles $(2 \mathrm{~S}, \mathrm{P})$, four for the period- 2 cycles $(2 \mathrm{P}, \mathrm{T}+\mathrm{S})$, and six for the period-3 cycle $(2 \mathrm{P}+2 \mathrm{~S})$. The period-1 frequency of the trajectories is also determined by the vortex dynamics. At $r / L=0.5, \phi_{s}^{*}=0$, and the cross stream width is $w=L / 2$, which results in the standard bluff body Strouhal number $S t=f w / U \simeq 0.2$. As $r / L$ is decreased, the fixed points spread from $\phi=0$ increasing the effective cross stream width, accounting for the decreased period-1 frequency observed in Fig 3.

Non-periodic behaviour is found between most of these periodic limit cycles for $r / L<$ 0.3. Figure 4 shows the response for selected trajectories which display many types of non-periodic behaviour associated with bi-stable oscillators. The trajectory at $r / L=0.23$ transition from one limit cycle to another (the $r / L=0.3$ cycle to the $r / L=0.25$ cycle). $r / L=0.19$ shows an extended non-periodic phase followed by stable period- 1 motion and the fixed point chosen is sensitive to the initial conditions. The other three $(r / L=0.21$, 0.185 , and 0.05 ) shows windows of periodic behaviour interspersed with non-periodic bursts of activity. Many of these trajectories feature non-periodic transitions from one of the stable fixed points to the other which results in a broadband frequency response.

A strong fully chaotic response is found in the window between the large period-3 orbit at $r / L=0.18$ and period-2 orbit at $r / L=0.1$. The chaotic response for $r / L=0.16$ is examined in detail in Fig 5. The time history is shown for a set of trajectories with a less than $1 \%$ distance between their initial conditions. Figure $5 \mathrm{~b}$ shows the average evolution of the distance between trajectories. The solutions remain close for times $t U / L<5$ while the flow around the cylinder initially develops. As the wake brakes symmetry the distance between the trajectories grows exponentially, with a (largest) Lyapunov exponent of 0.9. By $t U / L=12$ the trajectories have completely diverged. Figure $5 \mathrm{c}$ shows the phase portrait for one trajectory for $0 \leq t U / L \leq 10^{3}$. Similarities to the limit-cycle found at $r / L=0.18$ are evident, but most of the structural states within that envelope are visited at least once and the most common states are at $(\phi= \pm \pi / 4, \dot{\phi}=0)$ which is not in that limit cycle. Fig 5 also plots the normalized power spectral density of the trajectories. There is a peak at $f L / U=0.05$, which corresponds to period- 6 , but the response is generally broadband.

Figure 6 shows the vortex wake for the same chaotic case. In this figure, four times are shown when the trajectories pass through approximately the same point in the phase space. However, the wake in each image is completely unique; a lone pair of bound vor- 
(a)

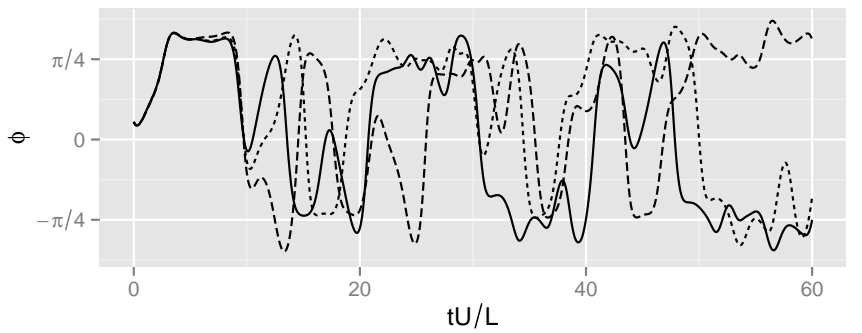

(c)

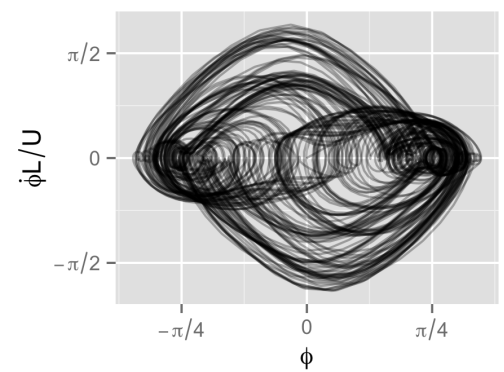

(d)

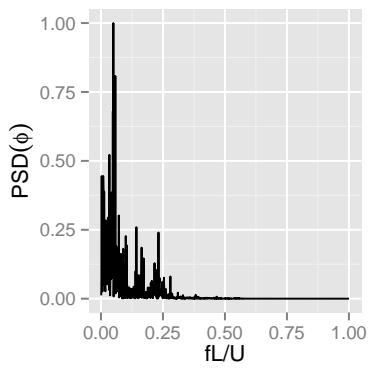

(b)

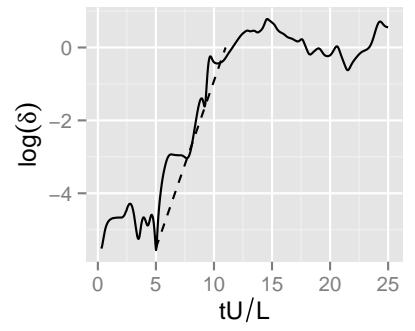

(e)

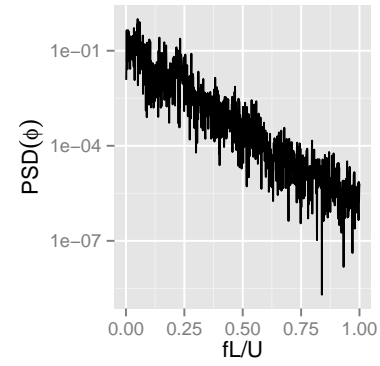

Figure 5: Trajectory characteristics of chaotic rotations with $r / L=0.16$ and $R e=1000$. (a) Time history of three trajectories started with initial conditions $\delta_{0}=.25 \%$ apart. (b) The evolution of the average distance between the trajectories in (a). The slope of the dashed line is 0.9 for reference. (c) Phase portrait for one trajectory from $0<t U / L<10^{3}$ using $70 \%$ line transparency. (d) Linear and (e) log plot of the normalized power spectral density of $\phi$ for the same trajectory as in (c).
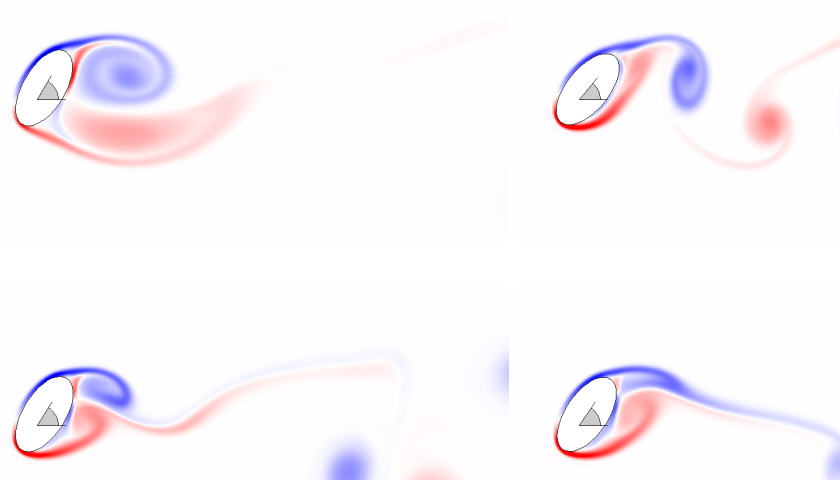

Figure 6: Vorticity field (red/blue) in the wake of the ellipse (black outline, travelling right-to-left) as it rotates freely in chaotic motion with $r / L=0.16$ and $R e=1000$. Four times are shown at approximately the same values of $\phi$ and $\dot{\phi}$. 
(a)

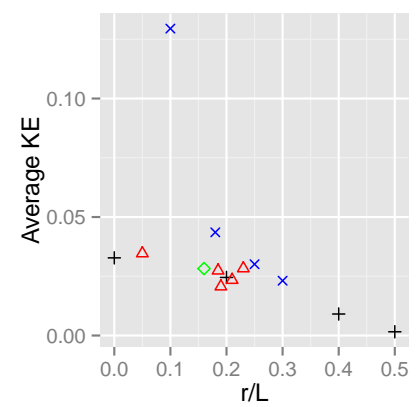

(b)

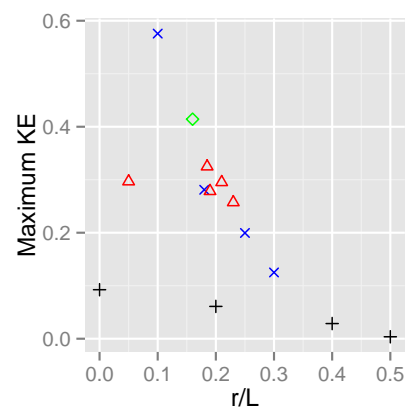

(c)

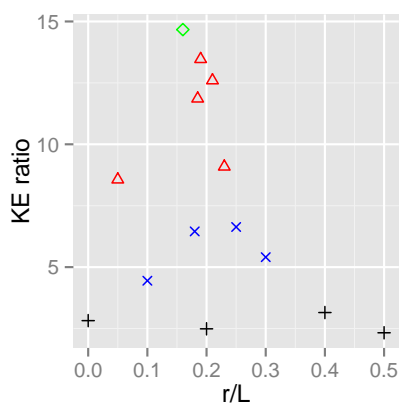

Figure 7: (a) Average and (b) Maximum rotational kinetic energy $\frac{1}{2} I_{0} \dot{\phi}^{2}$ of the ellipse scaled by the constant translational kinetic energy. (c) Ratio of the maximum to the average energy. Black crosses: period-1 cases, Blue x's: higher period limit cycles, Red triangles: intermittent cases, Green diamond: chaotic $r=0.16 L$ case. $R e=1000$.

tices, a set of $2 \mathrm{P}+\mathrm{S}$ vortices, and a large vortex pair shooting far off centerline upwards or downwards. The torque applied to the ellipse is unique in each case, leading to completely different future trajectories.

The rotational kinetic energy of the ellipse during free oscillations is shown in Fig 7 as a function of $r / L$. The trend is an increase in KE with decreasing $r / L$ until the $r / L=0.1$ cycle is reached, which has the maximum value observed. However, the details are markedly different for the different types of trajectories. The limit cycle cases show average energy levels that follow the trend mentioned, but the period-1 limit cycles have very low maximum energy levels. In contrast the average kinetic energies of the nonperiodic cases are essentially constant, but their maximum energy level increases with the trend. Plotting the ratio of maximum to average rotational kinetic energy cleanly divides the period-1 cycles below 3, the non-periodic orbits above 7 , and the higher period orbits between. The $r / L=0.16$ chaotic case has the maximum ratio of nearly 15 .

Finally, the dependence on damping is investigated in two ways; first, through the reduction of Reynolds number, and second, through the introduction of linear structural damping. Figure 8 shows the behaviour of the system as the Reynolds number is reduced for the highest energy trajectories, $0.1 \leq r / L \leq 0.16$. The increased fluid damping reduces the size and speed of oscillations throughout this range, and at $R e=100$ all trajectories have been damped to period-1 limit cycles around a single fixed point. However, there are still strong non-periodic response found for Reynolds numbers as low at 200. The change in Reynolds number also adjusts the location of the peak chaotic response from $r / L=0.16$ (as in Fig $5 \mathrm{c}$ ) down to $r / L \sim 0.1$. This is likely due to a change in the balance of the magnitude of the Munk moment and drag induced moment, quantified by the parameter $\beta$ in Eq 2.5. The increased fluid damping has also stabilized higher period responses than were observed at $R e=1000$, with the most extreme example being a period-14 limit cycle found at $R e=200, r / L=0.12$.

Figure 9 shows the result of adding linear structural damping torque $\zeta \dot{\phi}$ to the system, as in Eq 2.6. This is for the case of $r / L=0.16$ and $R e=1000$ which is chaotic for the undamped system. Here, the damping is nondimensionalized by $I_{0} f_{1} / \pi$ where $f_{1} L / U=0.3$ is the observed period- 1 frequency in this range. Therefore, $\zeta=1$ corresponds to critical damping of this frequency in the absence of fluid excitation. As with the Reynolds num- 

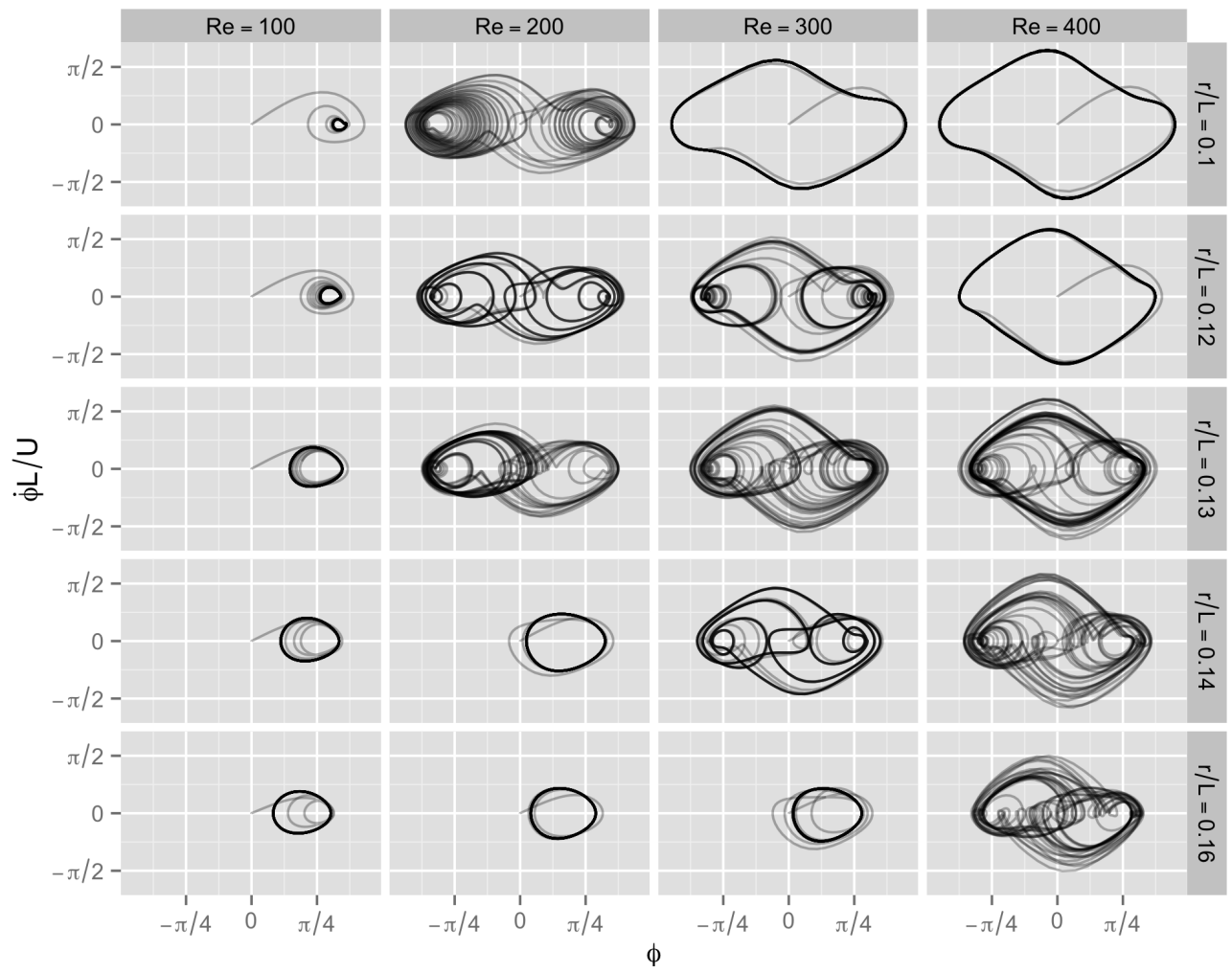

Figure 8: Phase portraits for $R e=100-400$ and $r / L=0.1-0.16$.

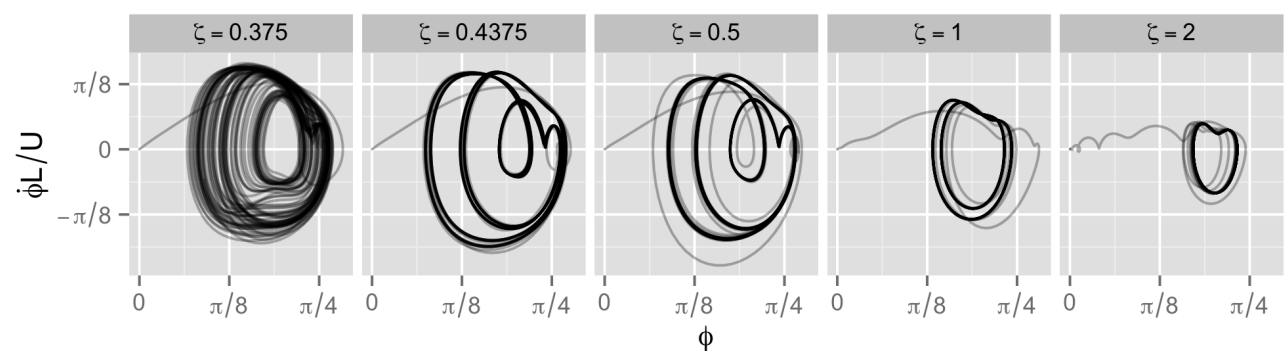

Figure 9: Phase portraits for $\zeta=0.375-2$ with $R e=1000$ and $r / L=0.16$.

ber study, adding damping results in a less energetic trajectory, and $\zeta=2$ (overdamped) results in a period-1 limit cycle around the stable fixed point. Less extreme damping destabilizes this trajectory and leads to period- 2 cycle at $\zeta=1$, period- 3 at $\zeta=0.5$, and a period- 6 cycle at $\zeta=0.4375$. By $\zeta=0.375$ the trajectory is fully chaotic but much less energetic than the undamped case, with velocities $1 / 4$ the magnitude and no switching between the positive and negative fixed points. 


\section{Discussion and Conclusions}

This work considers a simple towed elliptical cylinder which is free to rotate around a pivot, and demonstrates that the system responds with a rich set dynamics, including limit cycles, period doubling, and chaotic motions. The mean restoring torque was approximated analytically and used to show that the system has a region of natural bi-stability dependant on the distance $r / L$ between the centroid and the pivot. The underlying system is closely related to that of the classic forced-bistable oscillators, which are well known to incorporate these dynamical behaviours.

The trajectories the limit cycles cases (Fig 3) are dominated by the vortex dynamics, as observed in the literature of autorotation (Lugt 1983). The frequency and number of oscillations in each limit cycle corresponds directly to the bluff body Strouhal number, indicating that this is the dominant time-scale in the system. Decreasing $r / L$ from 0.2 to 0.1 changes the character of the wake from trailing edge to leading edge vortex shedding with a corresponding increase in the amplitude of motion (Fig 2) and the occurrence of trajectories visiting both of the stable fixed-points.

The vortex wake of the chaotic case at $r / L=0.16$ is only weakly correlated with the state of the ellipse, featuring any number of vortices as well as pairs of vortices shooting far off center-line in either direction (Fig 6). This sensitive wake history effect is a necessary condition for chaotic motions and stands in sharp contrast to the period-1 oscillations before the Hopf bifurcation in which the wake is fully determined by the instantaneous state of the ellipse. The irregularity of the wake also illustrates the limits of the analogy to the classic forced bi-stable systems such as Holmes (1979). The current system is self-exciting, not subject to a harmonic forcing. Therefore there is no fixed frequency or amplitude of forcing, and a constant period Poincare map is no better at clarifying the strange attractor than a random sample of the phase portrait.

The $r / L=0.1$ period-2 limit cycle has the largest rotational energy observed in this study, with a maximum of nearly $60 \%$ of the translational kinetic energy. The ratio of maximum to average kinetic energy perfectly separates the trajectory types, consistent with the cascade of energy into a wider frequency spectrum. The ratio for the chaotic case is nearly 15 , indicating extremely nonlinear loading on the ellipse in this trajectory. This is consistent with the 'snap' loading observed in chaotic FSI problems with flexible bodies (Sipcic 1990; Connell \& Yue 2007).

The energy of the ellipse motions is reduced as the damping losses are increased either through structural damping or decreased Reynolds number. In this way it is possible to restrict even the highest energy cases $(0.1 \leq r / L \leq 0.16)$ to low-amplitude period-1 limit cycles. Fluid damping is seen to stabilize the motion of the system, and at $R e=200 \mathrm{a}$ period-14 limit cycle is observed, as well as fully non-periodic motion. Similarly, moderate structural damping enables period-6 limit cycles, before finally degenerating to chaotic motion for $\zeta \leq 0.375$.

The damping studies demonstrate that the energy of the FSI system is a critical factor for the stability of low-period limit cycles. This can be extended to the undamped case by considering how the energy changes as $r / L$ is adjusted. As $r / L$ is reduced from 0.5 to 0 , the stable fixed points diverge from $\phi=0$ increasing the effective cross-stream width of the object. This has the combined effect of decreasing the bluff body shedding frequency and, crucially, of increasing the mean drag force on the tow point. The tow point is therefore doing more work on the FSI system, and the total KE must increase with the decrease in $r / L$. The system cannot accommodate this energy by increasing its rotation frequency because this is set from the Strouhal number. Nor can the trajectories visit $\phi$ much greater than the stable fixed point because of the extremely large restoring 
force beyond this point (Fig 1b). The only resolution is for the system to dump this increased energy into infrequent high-energy vortex shedding events. This leads to the observed period doubling, increased complexity of the wake, 'snap' loading, and the onset of chaos.

New applications such as sensors and energy extraction devices rely on the sensitive response of the structure to the flow. This work successfully demonstrates that even the simplest possible nonlinear FSI problem incorporates the full range of sensitive chaotic responses, and the range of possible dynamics only increases as variations to the Reynolds number, mass ratio, and cylinder cross-section are considered. As such, it may serve as a canonical example of nonlinear interactions, suitable for education, detailed analytic and experimental analysis, and to instigate new passive or minimally-actuated hydrodynamic sensor and energy extraction designs.

\section{REFERENCES}

AbDelkefi, A, HAJJ, MR \& NAYFeh, AH 2013 Piezoelectric energy harvesting from transverse galloping of bluff bodies. Smart Materials and Structures 22 (1), 015014.

Alonso, G., Meseguer, J., Sanz-Andres, A. \& Valero, E. 2010 On the galloping instability of two-dimensional bodies having elliptical cross-sections. Journal of Wind Engineering and Industrial Aerodynamics 98 (8-9), 438 - 448.

Arrieta, AF, Hagedorn, P, Erturk, A \& Inman, DJ 2010 A piezoelectric bistable plate for nonlinear broadband energy harvesting. Applied Physics Letters 97 (10), 104102-104102.

Barrero-Gil, A., Alonso, G. \& Sanz-Andres, A. 2010 Energy harvesting from transverse galloping. Journal of Sound and Vibration 329 (14), 2873-2883.

Bearman, PW 1984 Vortex shedding from oscillating bluff bodies. Annual Review of Fluid Mechanics 16 (1), 195-222.

Beem, Heather, Hildner, Matthew \& Triantafyllou, Michael 2013 Calibration and validation of a harbor seal whisker-inspired flow sensor. Smart Materials and Structures 22 (1), 014012.

Bernitsas, Michael M, Rasghavan, Kamaldev, Ben-Simon, Y \& Garcia, EMH 2008 Vivace (vortex induced vibration aquatic clean energy): A new concept in generation of clean and renewable energy from fluid flow. Journal of offshore mechanics and Arctic engineering 130 (4).

Connell, B.S.H. \& Yue, D. K. P. 2007 Flapping dynamics of a flag in a uniform stream. Journal of Fluid Mechanics 581 (-1), 33-67.

Dahl, JM, Hover, FS, Triantafyllou, MS, Dong, S \& Karniadakis, GE 2007 Resonant vibrations of bluff bodies cause multivortex shedding and high frequency forces. Physical review letters 99 (14), 144503.

Holmes, Philip 1979 A nonlinear oscillator with a strange attractor. Philosophical Transactions of the Royal Society of London. Series A, Mathematical and Physical Sciences 292 (1394), 419-448.

HuAng, L 1995 Flutter of cantilevered plates in axial flow. Journal of Fluids and Structures 9 (2), 127-147.

Lorenz, Edward N. 1963 Deterministic nonperiodic flow. Journal of the Atmospheric Sciences 20 (2), 130-141.

Lugt, HANs J 1980 Autorotation of an elliptic cylinder about an axis perpendicular to the flow. Journal of Fluid Mechanics 99 (04), 817-840.

Lugt, Hans J 1983 Autorotation. Annual Review of Fluid Mechanics 15 (1), 123-147.

Modarres-Sadeghi, Y, Chasparis, F, Triantafyllou, MS, Tognarelli, M \& Beynet, $\mathrm{P} 2011$ Chaotic response is a generic feature of vortex-induced vibrations of flexible risers. Journal of Sound and Vibration 330 (11), 2565-2579.

NAKAmURA, Y 1990 Recent research into bluff-body flutter. Journal of Wind Engineering and Industrial Aerodynamics 33 (1), 1-10.

Obligado, M, Puy, M \& Bourgoin, M 2013 Bi-stability of a pendular disk in laminar and turbulent flows. Journal of Fluid Mechanics 728, R2. 
Robertson, I, Li, L, Sherwin, SJ \& Bearman, PW 2003 A numerical study of rotational and transverse galloping rectangular bodies. Journal of fluids and structures 17 (5), 681-699.

Sipcic, Slobodan R 1990 The chaotic response of a fluttering panel: The influence of maneuvering. Nonlinear Dynamics 1 (3), 243-264.

Spyrou, KJ \& Thompson, JMT 2000 The nonlinear dynamics of ship motions: a field overview and some recent developments. Philosophical Transactions of the Royal Society of London. Series A: Mathematical, Physical and Engineering Sciences 358 (1771), 1735-1760.

Townsend, Nicholas C \& Shenoi, RA 2013 Modelling and analysis of a single gimbal gyroscopic energy harvester. Nonlinear Dynamics pp. 1-16.

VAn Oudheusden, BW 1995 On the quasi-steady analysis of one-degree-of-freedom galloping with combined translational and rotational effects. Nonlinear Dynamics 8 (4), 435-451.

VAn Oudheusden, BW 1996 Rotational one-degree-of-freedom galloping in the presence of viscous and frictional damping. Journal of fluids and structures 10 (7), 673-689.

Weymouth, Gabriel D., Dommermuth, Douglas G., Hendrickson, Kelli \& Yue, Dick K.-P. 2006 Advancements in cartesian-grid methods for computational ship hydrodynamics. In 26th Symposium on Naval Hydrodynamics. Rome, Italy.

Weymouth, Gabriel D. \& Triantafyllou, M. S. 2013 Ultra-fast escape of a deformable jet-propelled body. Journal of Fluid Mechanics 721, 367-385.

Weymouth, Gabriel D. \& Yue, Dick K.-P. 2011 Boundary data immersion method for cartesian-grid simulations of fluid-body interaction problems. Journal of Computational Physics 230 (16), 6233-6247.

Wibawa, M. S., Steele, S. C., Dahl, J. M., Rival, D. E., Weymouth, G. D. \& TriANTAFYLLOU, M. S. 2012 Global vorticity shedding for a vanishing wing. Journal of Fluid Mechanics 695, 112-134.

Williamson, CHK \& Govardhan, R 2004 Vortex-induced vibrations. Annual Review of Fluid Mechanics 36, 413-455. 